\title{
In memoriam Ernst Kullmann, 1931 - 1996
}

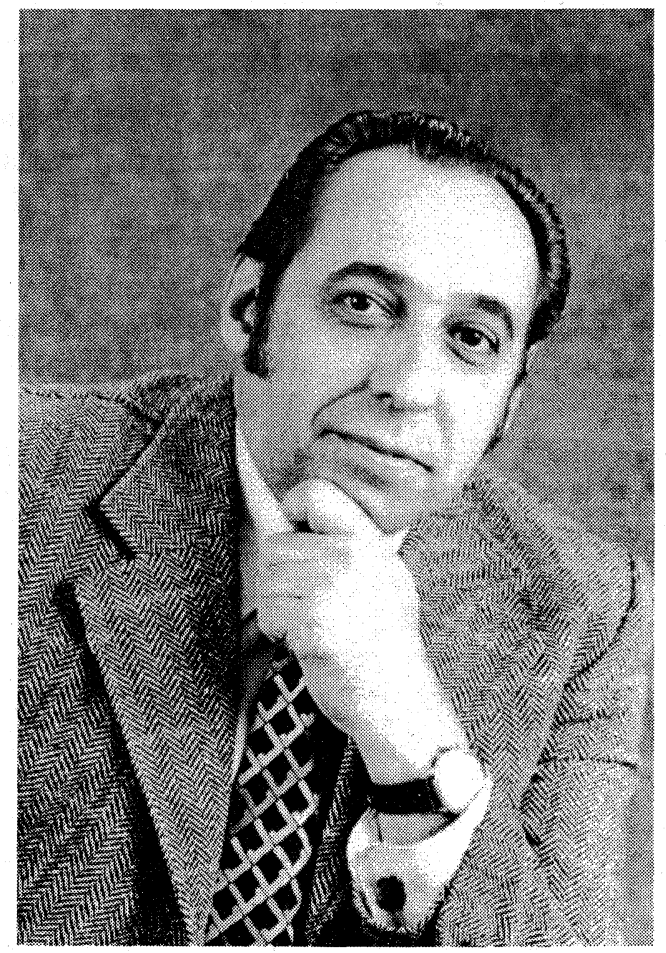

Die Arachnologie hat in der Person Ernst Kullmanns eines ihrer faszinierendsten Mitglieder verloren. Aber nicht nur die Arachnologie ist davon betroffen, es ist auch die Biologie im Allgemeinen, mitdem, was sie anziehender und menschlicher machte.

Es war anläßlich des IV. Internationalen Kongresses in Paris 1968, daß mir die Gelegenheit zuteil wurde, diesem außergewöhnlichen Mann, Ernst Kullmann, zu begegnen. Er hatte soeben eine wichtige Studie über die sardinischen Cyrtophoren beendet, nun präsentierte er seine ersten Arbeiten 
über Stegodyphus sarasinorum, eine afghanische Spezies. Überrascht darüber, daß wir beide über das Sozialverhalten der Spinnen forschten, begannen wir umgehend zusammenzuarbeiten, zunächst mit dem gemeinsamen Verzehr eines Pfeffersteaks, was tief in seiner Erinnerung eingemeißelt blieb, kam er doch noch des öfteren darauf zu sprechen.

Diese unbedeutend erscheinende Anekdote hat mir in der Tat das Wesen Ernst Kullmanns offenbart. Er war kontaktfreudig und begegnete den Menschen mit viel Respekt, ganz gleich ob Studenten oder Kollegen.

Das Bild indessen, das ich von ihm bewahre, ist vor allem das eines Biologen, der in erster Linie an der Biologie interessiert war und nicht an den Auszeichnungen, die sie ihm hier und da zuteil werden ließ. Von Modeerscheinungen unbeeinflußt, oft unkonventionell, die traditionellen Vorstellungen zurückweisend, die gelegentlich das Denken lähmen, bisweilen herausfordernd in der Absicht, die Leute zum Überdenken der "gängigen Vorstellungen" anzuhalten, hat er einen tiefen Eindruck im Bereich der Studien über die sozialen Merkmale der Spinnen hinterlassen und hatte keine Bedenken, die Radioaktive-Isotopen-Methode, die Histologie und die Elektronenmikroskopie mit der Ethologie zu verbinden.

Dennoch gab sich Ernst Kullmann nicht mit der Abgeschlossenheit seines Lebens zufrieden. Er war ein Mann, der auf jedem Terrain zu Hause war, sich um den Dialog bemühte, und der das Bedürfnis verspürte, sein biologisches Wissen an die Öffentlichkeit weiterzugeben. So verdanken wir inm eine Reihe bemerkenswerter Filme über Spinnen und einen weiteren über die "Krankheiten" der Erde (Ausbreitung der Wüsten, Abholzung der Wälder, Umweltverschmutzung usw.). Im selben Geiste übernahm er nach einem kurzen Gastspiel an der Universität Kiel die Leitung des Kölner Zoos. Er hatte zuvor den Zoo von Kabul gegründet. Die Eigenheit seiner Ideen und seine Tatkraft haben inm seine Aufgabe nicht leicht gemacht und nach ein paar Jahren gab er schließlich sein Amt auf, um sich von der Bühne zu verabschieden und in der Zurückgezogenheit zu arbeiten, was auch bedeutete, daß er an keinen internationalen Kolloquien über Arachnologie mehr teilnahm.

Leider erkennt man erst immer nach dem Tod die Qualitäten eines Menschens, und jene Ernst Kullmanns waren außergewöhnlich in Großmüt, Eigenheit und Tatkraft.

Betrand KRAFFT (Direktor Universität Nancy), Nancy, 14. Juni 1996 
$\mathrm{Zu}$ diesen Worten sollen nur einige kurze Bemerkungen hinzugefügt werden:

Als ein Verteter der neuen Arachnologen-Generation war mein erster Kontakt zu Ernst Kullmann ein indirekter über sein Buch „Leben am seidenen Faden", das er mit Horst Stern schrieb. Es hat mir - wie wahrscheinlich vielen anderen auch - die Arachnologie mit ihren vielgestaltigen und interessanten Facetten näher gebracht.

Später dann - beim Schreiben meiner Diplomarbeit - lernte ich Ernst Kullmann als einen hilfsbereiten und großzügigen Menschen kennen. Bei den wenigen Treffen und Spaziergängen durch die Wahner Heide bei Köln, die ich mit Ernst Kullmann erleben durfte, erzählte er oft aus der Vergangenheit und u.a. nicht selten von seiner Arbeit mit und Freundschaft $z u$ Hermann Wiehle und Wolfgang Crome. Dabeierschien er mir wie ein Bindeglied zwischen einer vergangenen Generation, die ich nur aus Literaturzitaten oder von Fotos kannte.

Ernst Kullmann wurde 1931 in Schneppenbaum bei Kleve geboren. Nach dem Abitur studierte er in Bonn Zoologie und Medizin. Er promovierte 1957 über den Netzbau bei Spinnen und habilitierte später in Zoologie und Parasitologie. 1962 bis 1966 weilte Ernst Kullmann im Rahmen einer Partnerschaft der Universitäten Bonn und Kabul in Afghanistan, wo er unter anderem den Kabuler Zoo aufbaute und zahlreiche Forschungsreisen unternahm. 1972 bis 1976 leitete er das Zoologische Institut in Kiel. 1975 wurde er zum Direktor des Kölner Zoos ernannt. Nach seiner frühzeitigen Pensionierung 1981 verfaßte er Reisebeschreibungen und unternahm nun privat Forschungsreisen. Von der letzten Reise aus Costa Rica kehrte er krank zurück und starb nach langem Fieber in Köln-Poll.

1960 rief Ernst Kullmann nach dem Zoologenkongreß in Bonn zusammen mit Wolfgang Crome, Hermann Wiehle und Otto Kraus das erste Spinnensymposium ins Leben, das zunächstjährlich stattfand. Durch diese Tatsache und die unersetzliche Öffentlichkeitsarbeit, die er mit seinem Buch und dem dazugehörigen Film (Bemerkungen über die Spinne) leistete, sind die Arachnologen inm zu großem Dank verpflichtet.

Peter JÄGER (Universität Mainz) 
Die Liste der Veröffentlichungen von Ernst Kullmann soll seine weitverzweigten wissenschaftlichen Interessen verdeutlichen. Die damit verbundenen Probleme der Vollständigkeit der Liste waren nur zum Teil alleine zu bewältigen. Zu danken ist seiner Frau Editha Kullmann und seinem Sohn Dr. Harald Kullmann (Köln), ferner Dr. V.Barus (Brno), Dr. Rainer Foelix (Aarau, Schweiz), Dr. Matthias Forst (Köln), Dr. Manfred Grasshoff (Frankfurt), Prof. Dr. Otto Kraus (Hamburg), Prof. Dr. Claas Naumann (Bonn), Dr. Vlastimil Ruzicka (Ceske Budejovice), Dorothee Sensen (Bochum), Dr. Konrad Thaler (Innsbruck) und Dr. Waltraud Zimmermann (Köln).

LEHMENSICK, R. \& E.KULLMANN (1956): Über den Feinbau der Fäden einiger Spinnen. - Proc. Stockholm Conference on Electron Microscopy: 307-309

LEHMENSICK, R. \& E.KULLMANN (1956): Über den Feinbau der Fäden einiger Spinnen. - Zool. Anz. (Suppl.) 19: 123-129

KULLMANN, E. (1958): Beobachtungen des Netzbaues und Beiträge zur Biologie von Cyrtophora citricola FORSKAL (Araneae, Araneidae). (Zugleich ein Beitrag zur Phylogenie der Radnetzspinnen). - Zool. Jb. Syst. 86: 181-216. Jena

KULLMANN, E. (1959): Beobachtungen und Betrachtungen zum Verhalten der Theridiide Conopistha argyrodes WALCKENAER (Araneae). Über das unterschiedliche Verhalten von Conopistha argyrodes gegenüber Cyrtophora citricola FORSKAL und Zygiella $x$-notata (THORELL). - Mitt. zool. Mus. Berlin 35 (2): 276-292

KULLMANN, E. (1959): Beobachtungen an der Raumnetzspinne Cyrtophora citricola FORSKAL auf Sardinien (Araneae, Araneidae). - Dtsch. entomol. Z. 6: 65-81

KULLMANN, E. (1959): Beobachtungen und Betrachtungen zum Verhalten der Theridiide Conopistha argyrodes WALCKENAER (Araneae). - Mitt. zool. Mus. Berlin 35 (2): 275-292 + 4 Taf.

KULLMANN, E. (1960): Beobachtungen an Theridium tepidariorum C. L. KOCH als Mitbewohner von Cyrtophora-Netzen (Araneae, Theridiidae). - Dtsch. entomol. Z. 7 (1/2): 146-163

KULLMANN, E. (1960): Über parasitäres Verhalten der Spinne Theridium tepidariorum C.L. KOCH. - Zool. Anz. (Suppl.) 23: 332-342

KULLMANN, E. (1961): Der Eierkokonbau von Cyrtophora citricola FORSKAL (Araneae, Araneidae). - Zool. Jb. Syst. 89: 369-406 + 6 Taf.

KULLMANN, E. (1961): Über das bisher unbekannte Netz und das Werbeverhalten von Drapetisca socialis (SUNDEVALL), (Araneae Linyphiidae). - Decheniana 114 (1): 99-104

KULLMANN, E. (1962): Über das eigenartige Deckennetz der Spinne Lepthyphantes obscurus (BLACKWALL, 1841) (Araneae, Linyphiidae). - Decheniana 114 (2): 105-109

KULLMANN, E. (1962): Über die vordringlichsten Aufgaben der Zoologischen Forschung und Lehre in Afghanistan. - Science (Afghanistan) 2 (2) [in persisch mit deutsch. Zusammenf.]

KULLMANN, E. (1963): Ein neues parasitologisches Problem in Afghanistan: Trichinella spiralis und die Trichinellosis. - Science (Afghanistan) 3 (1/2): 1-12 [in deutsch, Sonderheft in persisch] 
KULLMANN, E. (1964): Neue Ergebnisse über den Netzbau und das Sexualverhalten einiger Spinnenarten (Cresmatoneta mutinensis, Drapetisca socialis, Lithyphantes paykullianus, Cyrtophora citricola) als Beitrag zur Frage der Bedeutung besonderer Verhaltensmerkmale für die Systematik. - Z. zool. Syst. Evolutionsforsch. 2 (1/2): 41-122

KULLMANN, E. (1965): Über den ersten Nachweis von Trichinella spiralis (OWEN) in Afghanistan. - Z. Parasitenkde 25: 393-398

KULLMANN, E. (1965): Die Säugetiere Afghanistans. Teill: Carnivora, Artiodactyla, Primates. - Spec. Ed. Inst. Zool. und Parasit., Kabul Univ., Afghanistan: 1-17 [in German and Persian]

KULLMANN, E. (1967): Der Tiergarten Kabul - ein Zoo in statu nascendi. - Freunde d. Kölner Zoos 9: 130-134

KULLMANN, E. (1967): Wozu bauen wir einen Zoo in Kabul? - Freunde d. Kölner Zoos 10 (2): $43-49$

KULLMANN, E. (1967): Über das Verhalten der Hundebandwürmer in Afghanistan und dessen Bedeutung für die Epidemologie der Echinococcosis. - Zool. Anz. (Suppl.) 31: 204-216

KULLMANN, E. (1967/68): Über Leoparden Afghanistans und ihre Parasiten. - Freunde d. Kölner Zoos 10 (4): 126-135

KLOFT, W. \& E.KULLMANN (1968): Wie füttert die Haubennetzspinne Theridium notatum ihre Jungen? - Umschau Wiss. Techn. 23: 720-721

KULLMANN, E. (1968): Das Cribellum zweier Stegodyphus-Arten im elektronenoptischen Bild (Arachnida: Araneae: Eresidae). - Senckenbergiana biol. 49 (6): 451-460

KULLMANN, E. (1968): Soziale Phaenomene bei Spinnen. - Insectes Sociaux 15 (3): 289-298 KULLMANN, E. (1968): Als Zoologe in Afghanistan. - Tierschutznachr. Kölner Tierschutzverein KULLMANN, E. (1968/69): Expedition in die Heimat der Marco-Polo-Schafe - ein Beitrag über die Tierwelt des afghanischen Pamir. - Freunde d. Kölner Zoos 11 (4): 107-122

KULLMANN, E. \& W.KLOFT (1969): Traceruntersuchungen zur Regurgitationsfütterung bei Spinnen (Araneae, Theridiidae). - Zool. Anz. (Suppl.) 32: 487-497

KULLMANN, E. (1969): Soziales Verhalten bei Spinnen. - Mitt. phys.-med. Ges. Würzburg 77: $1-12$

KULLMANN, E. (1969): Spinnorgan mit 40000 „Düsen“. - Umschau Wiss. Techn. 3: 82-83, 3 fig.

KULLMANN, E. (1969): Unterschiedliche Brutfürsorge bei den Haubennetzspinnen Theridion impressum (L.KOCH) und Theridion notatum (CLERCK) (Araneae, Theridiidae). - Zool. Anz. (Suppl.) 33: 326-333, 3 fig.

KULLMANN, E. (1969): Brutpflege mit Regurgitationsfütterungen bei Haubennetzspinnen (Araneae, Theridiidae). Erläuterungen zu einem wissenschaftlichen Film. - Zool. Anz. (Suppl.) 33: 636-638

KULLMANN, E. (1969): Parasitologische Aspekte zur Viehwirtschaft der Nomaden. In: Nomadismus als Entwicklungsproblem, Bochumer Symposium 14./15. Juli 1967, Hrsg. vom Inst. Entwicklungsforsch. und Entwicklungspol. Ruhr-Universität Bochum. - Bochumer Schriften zur Entwicklungsforsch. und Entwicklungspol. Bd. 5, Bielefeld, Bertelsmann Universitätsverlag, S. 145-153

KULLMANN, E. (1970): Feststellungen zur Trichinellosis in der freien Wildbahn Afghanistans. - Proc. 2nd Int. Conf. on Trichinellosis, Wroclaw, Polen, Wiadomosci Parazytologiczne. 16: 11-116

BARUS, V., E.KULLMANN \& F.TENORA (1970): Neue Erkenntnisse über Nematoden und Acanthocephalen aus Nagetieren Afghanistans. - Acta soc. Zool. Bohem. 34: 236-276 
KULLMANN, E. (1970): Beobachtungen zum Sozialverhalten von Stegodyphus sarasinorum KARSCH (Araneae, Eresidae). - Bull. Mus. nat. hist. natur. Paris, (2e) 41 ( Suppl.1): 76-81

KULLMANN, E. (1970): Die Tierwelt Ostafghanistans und ihre geographischen Beziehungen. - Freunde d. Kölner Zoo 13 (1): 3-25

KULLMANN, E. \& F.TENORA (1970): Erste Nachweise von Bandwürmern aus Nagetieren (Rodentia) und Hasenartigen (Lagomorpha) Afghanistans. - Helmithologia 11 (1971) 1-4: 113-126

KULLMANN, E. \& F.TENORA (1970): Bandwürmer aus Insektenfressern (Insectivora) und Raubtieren (Carnivora) Afghanistans. - Helmithologia 11 (1971) 1-4: 127-139

KULLMANN, E. (1970/71): Bemerkenswerte Konvergenzen im Verhalten cribellater und ecribellater Spinnen. - Freunde d. Kölner Zoo 13 (4): 123-150

KULLMANN, E. \& S.NAWABI (1971): Versuche zur Trägerfunktion aasfressender Käfer (Silphidae, Carabidae) bei der Trichinellosis. - Z. Parasitenkde. 35 (3): 234-240

KULLMANN, E., H.SITTERTZ \& W.ZIMMERMANN (1971): Erster Nachweis von Regurgitationsfütterungen bei einer cribellaten Spinne (Stegodyphus lineatus LATREILLE, 1817, Eresidae). - Bonner zool. Beitr. 22 (1/2): 175-188

KULLMANN, E., S.NAWABI \& W.ZIMMERMANN (1972): Neue Ergebnisse zur Brutbiologie cribellater Spinnen aus Afghanistan und der Serengeti (Araneae, Eresidae). - Z. Kölner Zoo 14 (3): 87-108

BARUS, V., E.KULLMANN \& F.TENORA (1972): Parasitische Nematoden aus Wirbeltieren Afghanistans. - Acta Sci. Nat. Brno 6 (1): 1-46

KIRCHNER, W. \& E.KULLMANN (1972): Ökologische Untersuchungen an einer Freilandpopulation von Nesticus cellulanus im Siebengebirge unter besonderer Berücksichtigung der Kälteresistenz (Araneae, Nesticidae). - Decheniana 125 (1/2): 219-227

KULLMANN, E. (1972): The convergent development of orb-webs in cribellate and ecribellate spiders. - Amer. Zoologist 12: 395-405

KULLMANN, E. (1972): Evolution of social behaviour in spiders (Araneae, Eresidae and Theridiidae). - Amer. Zoologist 12: 419-426

KULLMANN, E. \& W.ZIMMERMANN (1973): Versuche zur Toleranz bei der permanentsozialen Spinnenart Stegodyphus sarasinorum KARSCH (Fam.: Eresidae). - Proc. 5th Int. Congr. Arachnology (Brno 1971) : 175-182. Brno

KULLMANN, E. (1973): Theridion impressum (Theridiidae). Brutfürsorge und periodischsoziales Verhalten. - Inst. für den wissensch. Film, Göttingen. Film E 1864 / 1973.

KULLMANN, E. (1974): Theridion sisyphium (Theridiidae). Brutfürsorge und periodischsoziales Verhalten. - Inst. für den wissensch. Film, Göttingen. Film E 1865 / 1973.

KIRCHNER, W. \& E.KULLMANN (1975): Überwinterung und Kälteresistenz der Haubennetzspinnenarten Theridion impressum (L.KOCH) und Theridion sisyphium (CLERCK) (Araneae, Theridiidae). - Decheniana 127: 241-250

KULLMANN, E. \& W. ZIMMERMANN (1975): Regurgitationsfütterungen als Bestandteil der Brutfürsorge bei Haubennetz- und Röhrenspinnen (Araneae, Theridiidae und Eresidae). - Proc. 6th Int. Congr. Arachnology (Amsterdam 1974) : 120-124, 1 Plate. Amsterdam

KULLMANN, E., W.BÖCKELER \& K.BUNGARD (1975): Feststellungen an heimischen Käfern als experimentellen Transitwirten von Trichinella spiralis (OWEN, 1835). - Zool. Anz. 194 (3/4): 180-192

KULLMANN, E., F.OTTO, T.BRAUN \& R.RACCANELLO (1975): Grundlagen und Ordnung - Übersicht der Netzkonstruktionen der Spinnen. - In: Netze in Natur und Technik. Mitt. Inst. leichte Flächentragwerke (IL) Univ. Stuttgart, 8: 304-316 
KULLMANN, E. (1975): Fäden und Netze von Spinnen und Insekten. Die Produktion und Funktion von Spinnenfäden und Spinnengeweben. - In: Netze in Natur und Technik. Mitt. Inst. leichte Flächentragwerke (IL) Univ. Stuttgart, 8: 318-378.

KULLMANN, E. \& H.STERN (1975): Leben am seidenen Faden. Die rätselvolle Welt der Spinnen. 300 S., München, Bertelsmann

STERN, H., K.HIRSCHEL \& E.KULLMANN (1975): Leben am seidenen Faden. 1. + 2. Film, Südfunk Stuttgart.

KULLMANN, E. (1976): Leben am seidenen Faden. Erläuterung zur Sonderausstellung lebender Spinnentiere und zahlreicher Fotographien, insbesondere rasterelektronenmikroskopischer Aufnahmen - Ausstellungszeit: 7. Januar bis 27. Februar 1977.

- Zeitschrift d. Kölner Zoos 19 (4): 137-144

KULLMANN, E. (1976): Jahresbericht 1975 der Aktiengesellschaft Zoologischer Garten Köln. - Zeitschrift d. Kölner Zoos 19 (2): 31-46

KULLMANN, E. \&W.ZIMMERMANN (1976): Ein neuer Beitrag zum Cribellaten-EcribellatenProblem: Beschreibung Uroecobius ecribellatus. $n$. gen. $n$. sp. und Diskussion seiner phylogenetischen Stellung (Arachnida: Araneae: Oecobiidae). - Ent. germ. 3 (1/2): 29-40

KULLMANN, E. \& W.ZIMMERMANN (1976): Beschreibung der neuen Spinnenart Oecobius afghanicus mit ergänzenden Angaben zu Oecobius putus und Oecobius annulipes (Arachnida: Araneae: Oecobiidae). - Ent. germ. 3 (1/2): 41-50

KULLMANN, E. (1977): Jahresbericht 1976 der Aktiengesellschaft Zoologischer Garten Köln. - Zeitschrift d. Kölner Zoos 20 (3): 71-98

KULLMANN, E. (1978): Jahresbericht 1977 der Aktiengesellschaft Zoologischer Garten Köln. - Zeitschrift d. Kölner Zoos 21 (1): 3-23

KULLMANN, E. (1978/79): Ein Nachwort zur Ausstellung "Insekten und Spinnen aus Edelstahl und in Zeichnungen von Hans JÄHNE". - Zeitschrift d. Kölner Zoos 21 (3): 98 KULLMANN, E. (1979): Dr. Wilhelm WINDECKER. - Zeitschrift d. Kölner Zoos 21 (4): 109 KULLMANN, E. (1979): Jahresbericht 1978 der Aktiengesellschaft Zoologischer Garten Köln. - Zeitschrift d. Kölner Zoos 22 (3): 75-91

KULLMANN, E. (1979): Eine neue Art von Außenkäfigen für Varis (Lemurv. variegatus) und Guerezas (Colobus guereza kikuyensis). - Zeitschrift d. Kölner Zoos 22 (3): 103-107

KULLMANN, E. (1979): Die Todeslawine - Unsere Umwelt und ihre Bedrohung durch den Menschen - 10-teilige Filmserie des WWF (Westdeutsches Werbefernsehen)

KULLMANN, E. (1980): Jahresbericht 1979 der Aktiengesellschaft Zoologischer Garten Köln. - Zeitschr. d. Kölner Zoos 23 (3): 79-101

KULLMANN, E. (1982): Natur und Mensch: Die Todeslawine - Unsere Umwelt und ihre Bedrohung durch den Menschen. I. - Der Samariter (Mitgliederzeitschrift des ArbeiterSamariter-Bundes) 2: 16-17

KULLMANN, E. (1983): Natur und Mensch: Die Todeslawine - Unsere Umwelt und ihre Bedrohung durch den Menschen. II. - Der Samariter (Mitgliederzeitschrift des ArbeiterSamariter-Bundes) 1: 28-29

KULLMANN, E. (1983): Natur und Mensch: Die Todeslawine - Unsere Umwelt und ihre Bedrohung durch den Menschen. III. - Der Samariter (Mitgliederzeitschrift des ArbeiterSamariter-Bundes) 2; 33-34

KULLMANN, E. (1983): Natur und Mensch: Die Todeslawine - Unsere Umwelt und ihre Bedrohung durch den Menschen. IV. - Der Samariter (Mitgliederzeitschrift des ArbeiterSamariter-Bundes) 3: 32-33 
KULLMANN, E. (1983): Natur und Mensch: Die Todeslawine - Unsere Umwelt und ihre Bedrohung durch den Menschen. V. - Der Samariter (Mitgliederzeitschrift des ArbeiterSamariter-Bundes) 4: 48-49

KULLMANN, E. (1988): Netzbau und Beutefangverhalten bei der Sektorspinne. - In: Pareys Studientexte 61: Praktische Verhaltensbiologie. Verlag Paul Parey-Berlin und Hamburg, S. 83-92

KULLMANN, E. (1990): Spinnen - wenig bekannte, meist verkannte Wesen um uns. - Mennoblatt (Paraguay) 10: 7-8

KULLMANN, E. (1990): Spinnen - hochentwickelte Geschöpfe. - Mennoblatt (Paraguay) 19: 6-8

KULLMANN, E. (1991): Über eine Sozialspinne aus dem Gran Chaco: Eriophora socialis (RENGGER, 1836). - Tier u. Museum 2 (4): 105-112

KULLMANN, E. (1993): Spinnen: Haben Sie ihnen schon einmal ins Auge geblickt? - „Sechzig - Na und?" Bonn-Bad Godesberg 7 (3): 12-16

KULLMANN, E. (1993): Madagaskar - Inselkontinent der „halben“ Affen. - „Sechzig - Na und?" Bonn-Bad Godesberg 7 (4): 36-41

KULLMANN, E. (1993): Die Lungen unserer Erde werden zu Staublungen. - „Sechzig $\mathrm{Na}$ und?" Bonn-Bad Godesberg 7 (5): 37-41

KULLMANN, E. (1993): Wüsten - Ein Landschaftstyp mit Zukunft. - „Sechzig - Na und?" Bonn-Bad Godesberg 7 (6): 36-40

KULLMANN, E. (1994): Das Herz Südamerikas: Paraguay. - „Sechzig - Na und?" Bonn-Bad Godesberg 8 (1): $36-41$

KULLMANN, E. (1994): Das Herz Südamerikas: Paraguay. Hier: Zur Flora und Fauna. - „Sechzig - Na und?" Bonn-Bad Godesberg 8 (2): 38-42

KULLMANN, E. (1994): Costa Rica - Ein Tropenland, in das man sich verlieben kann. - „Sechzig - Na und?" Bonn-Bad Godesberg 8 (3): 34-39

KULLMANN, E. (1994): Das Museum Alexander König in Bonn. - „Sechzig - Na und?" Bonn-Bad Godesberg 8 (4): 27-31

KULLMANN, E. (1994): Die Gentechnik: Zu ihrem Wesen, Nutzen und ihren Gefahren. - „Sechzig - Na und?" Bonn-Bad Godesberg 8 (4): 36-37

KULLMANN, E. (1994): Ein Besuch im Zoo. - "Sechzig - Na und?" Bonn-Bad Godesberg 8 (5): 38-42

KULLMANN, E. (1995): Nuristan- "Land des Lichts“. - „Sechzig - Na und?" Bonn-Bad Godesberg 8 (6): 34-39

KULLMANN, E. (1995): Ein wenig bekanntes Phänomen: „soziale“ Spinnen. Spinnen als Kommunarden. - „Sechzig - Na und?" Bonn-Bad Godesberg 9 (1): 38-42

KULLMANN, E. (1995): Die Bären: Mythologisch und zoologisch betrachtet. - „Sechzig Na und?" Bonn-Bad Godesberg 9 (2): 38-42

KULLMANN, E. (1995): Die Arche Noah - biblisch, technisch und zoologisch gesehen.

- „Sechzig - Na und?" Bonn-Bad Godesberg 9 (3): 34-39

KULLMANN, E. (1995): Eulen: Hier nicht nach Athen getragen. - „Sechzig - Na und?" Bonn-Bad Godesberg 9 (4): 34-37

KULLMANN, E. (1995): Die Falknerei: Der noble Umgang mit dressierten Greifen. - „Sechzig - Na und?" Bonn-Bad Godesberg 9 (5): 34-37

KULLMANN, E. (1996): Tiere als Götter und religiöse Symbole. - „Sechzig - Na und?" Bonn-Bad Godesberg 10 (1): 38-42

KULLMANN, E. (1996): Schlangen: Ihr Wesen und Mythos. - „Sechzig - Na und?" Bonn-Bad Godesberg 10 (2): 38-42 